\title{
Growth phase- and desiccation- dependent Acinetobacter baumannii morphology: an atomic force microscopy investigation
}

\author{
AUTHORS: Shadi Bashiri, ${ }^{a}$ Massimiliano Lucidi, ${ }^{b}$ Daniela Visaggio, ${ }^{a}$ Giulia Capecchi, ${ }^{a}$ Luca \\ Persichetti, ${ }^{a}$ Gabriella Cincotti, ${ }^{b}$ Paolo Visca, ${ }^{a}$ and Giovanni Capellini ${ }^{a \#}$.
}

\author{
AFFILIATIONS: \\ a Department of Science, University Roma Tre, viale Guglielmo Marconi 446, 00146 Rome, Italy \\ ${ }^{\mathrm{b}}$ Department of Engineering, University Roma Tre, via Vito Volterra 62, 00146 Rome, Italy
}

Supporting Information

\begin{abstract}
Acinetobacter baumannii has emerged as a major bacterial pathogen during the past three decades. The majority of the $A$. baumannii infections occur in hospitals and are caused by strains endowed with high desiccation tolerance, which represents an essential feature for the adaptation to the nosocomial environment. This work aims at investigating the desiccation response of the multidrug-resistant A. baumannii strain ACICU as a function of the bacterial growth phase and oxygen availability, by correlating bacterial survival with shape alterations. The three dimensional morphological analysis of bacteria was carried out by atomic force microscopy (AFM), following the evolution of bacterial shape descriptors, such as the area, volume, roughness of individual cell membranes, and the cell cluster roughness, which exhibited peculiar and distinctive behavior as a function of the growth conditions. AFM images of A. baumannii ACICU cells revealed a prevalence of the coccoid morphology at all growth stages, with a tendency to reduce their size in the stationary phase, accompanied by a higher survival rate to air-drying. Moreover, cells harvested from the logarithmic phase featured a larger volume and resulted to be more sensitive to desiccation compared to the cells harvested at later growth stages. In addition, oxygen deprivation caused a significant decrease in cellular size and was associated with the formation of pores in the cell membrane, accompanied by a relative reduction in culturability after desiccation. Morphological plasticity and multidrug resistance may contribute to desiccation tolerance and therefore to persistence in the hospital setting.
\end{abstract}

KEYWORDS: Acinetobacter, Atomic Force Microscopy, bacteria, desiccation, morphology, nanoscopy, super-resolution microscopy.

\section{INTRODUCTION}

Bacterial species exhibit an extraordinary variability of shapes that are preserved through countless generations (1). Although the bacterial shape is genetically determined, sporadic changes in bacterial cell morphology can occur in response to environmental conditions (i.e., physical and chemical stressors, changes in osmolarity, colonization of other organisms, nutrient depletion or abundance, etc). Indeed, shape drives the interactions between a bacterial cell and its environment, and morphology modifications reflect the bacterial response to a given environmental change $(2$, 3).

The ability of adapting the morphology in response to the stress induced by antimicrobials has been reported for Acinetobacter baumannii $(4,5,6)$. A. baumannii is an opportunistic human pathogen responsible for a variety of hospital-acquired infections, especially among severely ill and immunocompromised patients in intensive care units $(7,8)$. In recent years, this bacterium has become a growing global concern due to the rapid emergence of multidrug-resistant strains (MDR) in a number of healthcare settings $(8,9)$. Among Gram-negative bacteria, A. baumannii is characterized by an impressive ability to tolerate long-term desiccation $(10,11,12)$ and to resist various temperatures, $\mathrm{pH}$ levels, and nutrient-limiting conditions, both on biotic and abiotic surfaces (13). For this peculiar feature, A. baumannii represents a model organism for desiccation studies in Gram-negative bacteria $(14,15,16,17)$, and to investigate the key features that promote survival and transmission of nosocomial pathogens $(11,12)$. Desiccation tolerance varies among A. baumannii strains: in particular, isolates adapted to the hospital environment generally appear more resistant than laboratory-adapted strains (12). In this work, the prototypic MDR A. baumannii strain ACICU (18) has been selected as a clinical isolate endowed with high desiccation resistance (12), and as a model organism to investigate changes in bacterial cell morphology due to desiccation.

To date, scanning and transmission electron microscopy have widely been used to investigate bacterial cell morphology or bacterial appendages (such as pili, membrane, flagella). These techniques reach the necessary resolution to allow visualization of bacterial microdomains at the nanoscale level. However, they have several limitations, including harsh chemical procedures in sample preparation and the necessity to work under vacuum and/or 
cryogenic conditions $(19,20)$. In recent years, atomic force microscopy (AFM) has brought novel insights into the assembly, dynamics, and functions of bacterial cell nanostructures thanks to its capability to return morphological information of the sample in three dimension (21 - 24). The high lateral and vertical resolution of the technique have enabled to reveal a wealth of ultrastructural features that are invisible to traditional, diffraction-limited optical microscopy techniques or imperceptible in their true physiological state by electron microscopy $(25,26)$.

Several aspects of $A$. baumannii have been investigated using AFM, such as the effects of antimicrobial compounds (4, 5, 27), the role of a transcriptional regulator involved in pathogenicity modulation (28), the mechanism of phage infection $(29,30)$, and the bacterial adhesion and biofilm formation (31).

In the present work, AFM was employed to study the $A$. baumannii ACICU morphology in different growth phases and culture oxygenation level. The A. baumannii ACICU cell viability was also determined at each condition in order to correlate survival to desiccation with morphometric parameters measured with AFM operating in Peak Force mode.

We demonstrated the correlation between quantitative cell morphology parameters extracted from AFM imaging and biologically relevant factors, such as the bacterial survival rate in the different growth phase/conditions, obtaining useful information for understanding the specific response of $A$. baumannii strains to environmental stresses.

\section{- EXPERIMENTAL SECTION}

Growth conditions. A. baumannii ACICU was grown in LB liquid medium (32) for $18 \mathrm{~h}$ at $37^{\circ} \mathrm{C}$ with vigorous shaking (250 rpm), then sub-cultured (dilution $1: 100$ ) in a $15-\mathrm{ml}$ tube containing $5 \mathrm{ml}$ of LB or in a $350 \mathrm{ml}$ flask containing $50 \mathrm{ml}$ of LB, and incubated at $37^{\circ} \mathrm{C}$ in static conditions (static tube; ST), or under vigorous aeration by rotary shaking at 250 revolutions/min (shaken flask; SF), respectively. The dissolved oxygen levels were $10 \%$ and $25 \%$ in ST and SF, respectively, as determined by using a Dissolved Oxygen Measuring System (Instech). Bacterial growth was monitored during time, measuring the absorbance at $600 \mathrm{~nm}$ $\left(\mathrm{OD}_{600}\right) \quad$ using a $\quad$ BioSpectrometer ${ }^{\circledR}$ (Eppendorf) spectrophotometer.

Viable cell counts. At each time point specified in the Results section, $1 \mathrm{ml}$ of bacterial culture was collected, washed twice, and diluted in distilled water to reach $\mathrm{OD}_{600}=1$, corresponding to $\mathrm{ca}$ $5 \times 10^{8}$ CFUs (Colony Forming Units) per ml. An aliquot of $20 \mu 1$ of the bacterial suspension was used to determine the CFU number by the plate dilution method, and a second aliquot of $20 \mu 1$ was spotted on a glass coverslip (Corning ${ }^{\circledR}$ cover glasses, Sigma-Aldrich) and air-dried under the laminar flow hood for $20 \mathrm{~min}$ at room temperature. Cell viability after desiccation was determined by viable counts on LB-agar (LA) plates before and after air-drying, as previously described (12). Briefly, desiccated samples were rehydrated in $2 \mathrm{ml}$ of distilled water, incubated for 15 minutes at room temperature, mixed by vigorous shaking for 30 seconds to ensure a complete detaching of bacteria from the glass coverslip, and appropriately diluted in saline (isotonic) solution for CFU counts. Statistical analysis was performed with the software GraphPad Instat (GraphPad Software, Inc., La Jolla, CA), using One-Way Analysis of Variance (ANOVA).
Sample preparation and AFM measurement. Bacterial cells were washed twice and diluted in distilled water to $\mathrm{OD}_{600}=1$. Twenty $\mu 1$ of the bacterial suspension was poured on a microscope glass slide (Thermo Scientific ${ }^{\mathrm{TM}}$ SuperFrost ${ }^{\mathrm{TM}}$ Microscope slides $76 \times 26 \mathrm{~mm}$, with $1 \mathrm{~mm}$ thickness) and air-dried under the laminar flow hood at room temperature for $20 \mathrm{~min}$. Glass slides were cleaned with pure ethanol before bacteria deposition. AFM measurements were performed using a Dimension ICON AFM (Bruker, Santa Barbara, USA) set to Peak Force mode equipped with a ScanAsyst-Air Bruker silicon probe featuring a nominal cantilever elastic constant of $0.4 \mathrm{Nm}^{-1}$ and a tip with a nominal radius of $2 \mathrm{~nm}$. The AFM images were recorded at a temperature range of $20-24{ }^{\circ} \mathrm{C}$. The oscillation frequency and oscillation amplitude of the cantilever were set to $1 \mathrm{kHz}$ and $150 \mathrm{~nm}$, respectively. The force setpoint was optimized in the $15 \mathrm{nN}-25 \mathrm{nN}$ range. For each experiment, multiple large scans $\left(25 \times 25 \mu \mathrm{m}^{2}\right)$ were performed in different regions of the glass slide, using a constant $512 \times 512$ pixels size in each image. Individual bacterial clusters were imaged acquiring images in the range of size of $10 \times 10 \mu \mathrm{m}^{2}$ to $15 \times 15 \mu \mathrm{m}^{2}$ depending on the cluster size. High resolution images of individual cells were acquired with scan sizes ranging from $2 \times 2$ $\mu \mathrm{m}^{2}$ to $5 \times 5 \mu \mathrm{m}^{2}$, at image sizes up to $700 \times 700$ pixels. For each condition, at least 7 images at different scan sizes and lateral resolutions were obtained. For each measurement, height sensor and Peak Force error images were recorded simultaneously. The AFM images were analyzed and processed with the software Gwyddion (33). For visulalization of the topographic images, we used the Gwyddion adaptive color bar mapping. All images were first-order flattened and set to the constant value of height to facilitate their comparison. After leveling, a metrological analysis was performed on at least 30 different, randomly selected cells to measure their biophysical parameters. Briefly, cellular length (L) and width $(\mathrm{W})$ were referred to elongated and short horizontal axes, respectively. Cell height $(\mathrm{H})$ was determined based on the maximum height measured along the cell cross section. The reported height was calculated by averaging at least 30 bacterial cells for each condition. Cell volume (V) and surface area were obtained by Gwyddion grain-analysis algorithm after masking individual cells. The depth of the cell-membrane pores was considered from the highest protruding rim relative to the lowest concave edge. Surface roughness was obtained by measuring the root mean square deviation of surface heights from AFM images of clusters. For the evaluation of the cluster roughness, we averaged over at least three $2.5 \mu \mathrm{m} \times 2.5 \mu \mathrm{m}$ areas within the same bacterial cluster. For what the individual cell roughness analysis is concerned, we analyzed a surface area of $400 \mathrm{~nm} \times 400 \mathrm{~nm}$, after subtraction of the background due to the overall curvature of the cell membrane, i.e. we analyzed the "local surface roughness", averaging over at least forty different cell areas.

\section{- RESULTS AND DISCUSSION}

\section{Growth phase influences desiccation tolerance and cell morphology}

Previous findings demonstrated that desiccation tolerance in $A$. baumannii type strain ATCC $19606^{\mathrm{T}}$ is a growth phase-dependent phenomenon. Indeed, cells harvested during the logarithmic growth phase were more sensitive to dehydration than stationary phase cells $(34,16,17)$. However, in these studies the desiccation tolerance was not systematically correlated to the cell morphology in the different growth phases. Consequently, we investigated 
whether also the A. baumannii ACICU strain shows a growth phase-dependent susceptibility to desiccation, and whether a correlation exists between growth phase, bacterial survival, and cell morphology (35). A. baumannii ACICU was cultured in LB medium and the bacterial growth was monitored for up to $48 \mathrm{~h}$ in a shaken flask (SF), in order to identify the different growth phases. By measuring the absorbance at $600 \mathrm{~nm}$ over time, the following growth phases were defined: lag $\left(t_{\text {growth }}<1 \mathrm{~h}\right)$, early logarithmic $(1$ $\left.\mathrm{h}<\mathrm{t}_{\text {growth }}<4 \mathrm{~h}\right)$, late logarithmic $\left(4 \mathrm{~h}<\mathrm{t}_{\text {growth }}<6 \mathrm{~h}\right)$, stationary $(6$ $\mathrm{h}<\mathrm{t}_{\text {growth }}<32 \mathrm{~h}$ ), and death phase ( $\mathrm{t}_{\text {growth }}>32 \mathrm{~h}$ ) (see Fig. 1A). Concurrently, at $3,6,14,24$, and $48 \mathrm{~h}$ of growth, bacterial cells were washed and normalized to $\mathrm{OD}_{600}=1$. The bacterial survival rates were determined before and after air-drying and expressed as viable counts (Colony-Forming Units CFU; Fig. 1B). During incubation of bacteria at $37^{\circ}$ in $\mathrm{LB}$ medium, one $\log \mathrm{CFU}$ reduction was observed only at $48 \mathrm{~h}$, confirming the predicted death phase, while no reduction in bacterial viability was observed in earlier growth stages. As expected, desiccation of A. baumannii ACICU cells taken from the early logarithmic phase caused a significant decrease $(\mathrm{P}=0.0024)$ in survival compared with cells harvested from late logarithmic $(\mathrm{P}=0.0326)$, stationary $(\mathrm{P}=0.055)$ or death $(\mathrm{P}=0.75)$ phase.

In order to determine if the desiccation effect on cell viability is correlated with an alteration of cell morphology, we used AFM to image A. baumannii ACICU cells at each growth phase (Figure 1C). In all the investigated samples, we observed a tendency of the bacteria to aggregate in clusters, leaving only a few sparse individual cells, probably due to the driving force of the evaporating water, inducing the so-called "coffee-stain" effect (36). Interestingly, the height of A. baumannii ACICU cells in earlylogarithmic growth phase was higher than those measured in the other growth phases (Figure 1D). Furthermore, AFM height images were used to provide quantitative information on the cell surface roughness as a function of the bacterial growth phase (Figure 1C). The cluster surface roughness was measured for at least three independent areas in a $2.5 \times 2.5 \mu \mathrm{m}^{2}$ square comprising several $A$. baumannii ACICU cells. As shown in Figure 1D, we observed that the cluster roughness was significantly decreased from $56 \mathrm{~nm}$ in the logarithmic phase to $32 \mathrm{~nm}$ in the stationary phase.
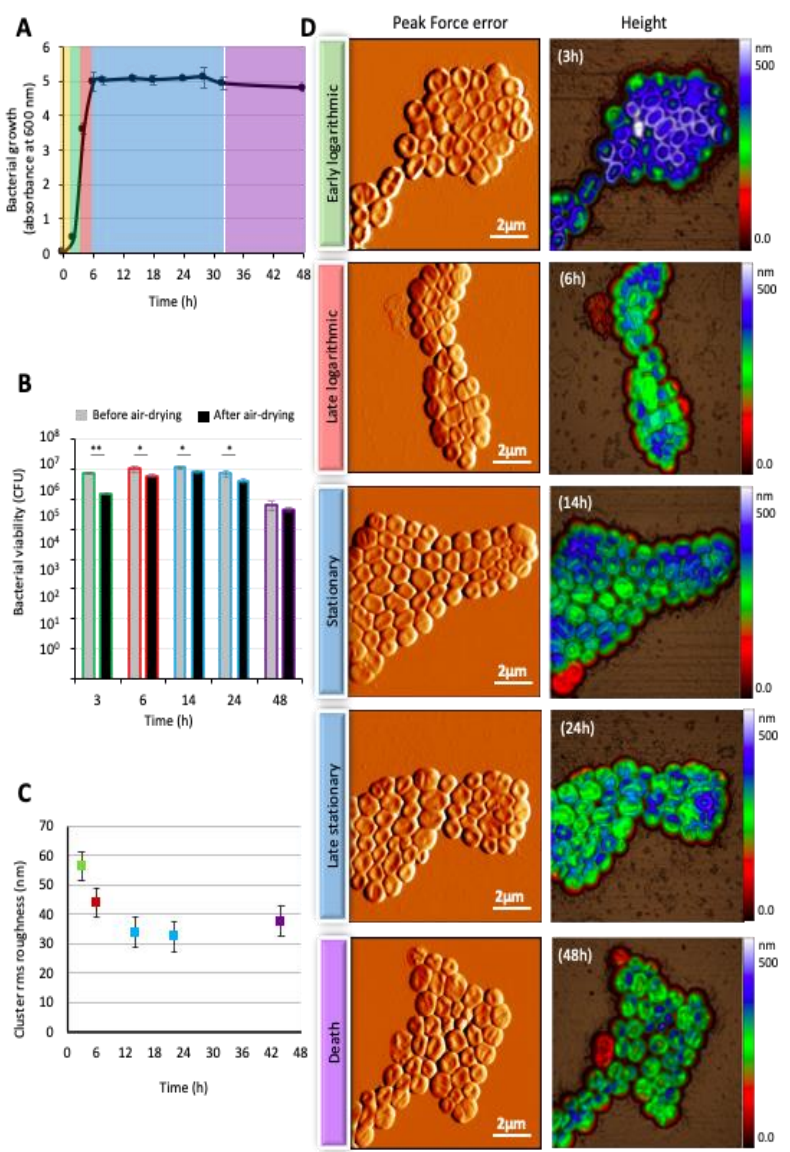

Figure 1. Growth, cell viability and morphology of $A$. baumannii ACICU during growth in shaken flasks (SF). (A) Growth plot of $A$. baumannii ACICU cultured in $\mathrm{LB}$ at $37^{\circ} \mathrm{C}$ under vigorous shaking. At the indicated time points, bacterial growth was measured as absorbance at $600 \mathrm{~nm}\left(\mathrm{OD}_{600}\right)$. The colored boxes yellow, green, red, cyan, and violet indicate lag, logarithmic, late logarithmic, stationary and death phases, respectively. (B) CFU counts at different growth phases before and after air-drying. Colors of the histogram bars outline correspond to the growth phases shown in panel A. (C) Roughness analysis of A. baumannii ACICU cell clusters was conducted over three different $2.5 \mu \mathrm{m} \times 2.5 \mu \mathrm{m}$ wide areas within the same bacterial cluster. The error is estimated as the mean absolute deviation around the mean. The color of the squares corresponds to the growth phases shown in panel A. (D) AFM Peak Force error and height images of A. baumannii ACICU cells at different growth phases. Asterisks indicate statistically significant differences between before and after air-drying conditions $(* \mathrm{P}<0.05 ; * * \mathrm{P}<0.01)$. 
To gain insight into the cell schape, the length, width, height, volume, and surface/volume ratio were calculated on more than 30 desiccated cells for each growth phase (Table 1). At all time points, A. baumannii ACICU cells displayed a coccoid morphology, with an oval surface projection featuring an average length-to-width ratio of $\mathrm{L} / \mathrm{W}=1.2 \pm 0.02$, suggesting that the shape of $A$. baumannii ACICU is not influenced by growth-phase under the tested conditions. We notice that, the majority of the A. baumannii ACICU cells is characterized by the presence of a height depression in the central part of the cell, which is typically $\sim 50 \mathrm{~nm}$ lower with respect to the cell edges. Although the overall cell morphology did not change, the height and the volume of cells in the earlylogarithmic phase were higher than those in the late logarithmic, stationary, late stationary, and death phase. Interestingly, $A$. baumannii ACICU cells in the stationary phase showed the lowest surface to volume ratio and were also the most resistant to desiccation, as compared with cells in the other growth phases. Therefore, we speculate that bacterial cells endowed with smaller surface are less exposed to water loss and consequently, their desiccation resistance is higher. This observation is in line with a previous study of Madigan and coworkers, demonstrating that smaller cells can tolerate adverse conditions better than the large cells $(37,38)$.

Table 1. Cellular dimensions at different growth phases of $A$. baumannii ACICU cultured in shaken flask (SF).

\begin{tabular}{cccccc}
\hline \multirow{2}{*}{ Growth phase } & \multicolumn{5}{c}{ Cellular dimensions } \\
\cline { 2 - 6 } & Length $(\boldsymbol{\mu m})$ & Width $(\boldsymbol{\mu m})$ & Height $(\boldsymbol{\mu m})$ & Volume $\left(\boldsymbol{\mu \mathbf { m } ^ { 3 } )}\right.$ & Surface/Volume $\left(\boldsymbol{\mu \mathbf { m } ^ { - 1 } )}\right.$ \\
\hline Logarithmic & $1.29 \pm 0.15$ & $1.08 \pm 0.11$ & $0.397 \pm 0.11$ & $0.350 \pm 0.06$ & $3.14 \pm 0.40$ \\
Late logarithmic & $1.06 \pm 0.14$ & $0.90 \pm 0.11$ & $0.335 \pm 0.04$ & $0.201 \pm 0.03$ & $3.79 \pm 0.61$ \\
Stationary & $0.96 \pm 0.17$ & $0.79 \pm 0.10$ & $0.325 \pm 0.04$ & $0.226 \pm 0.06$ & $3.02 \pm 0.31$ \\
Late stationary & $1.06 \pm 0.18$ & $0.88 \pm 0.12$ & $0.308 \pm 0.06$ & $0.203 \pm 0.05$ & $3.86 \pm 0.63$ \\
Death & $1.15 \pm 0.18$ & $0.96 \pm 0.13$ & $0.316 \pm 0.04$ & $0.260 \pm 0.04$ & $4.01 \pm 1.12$ \\
\hline
\end{tabular}

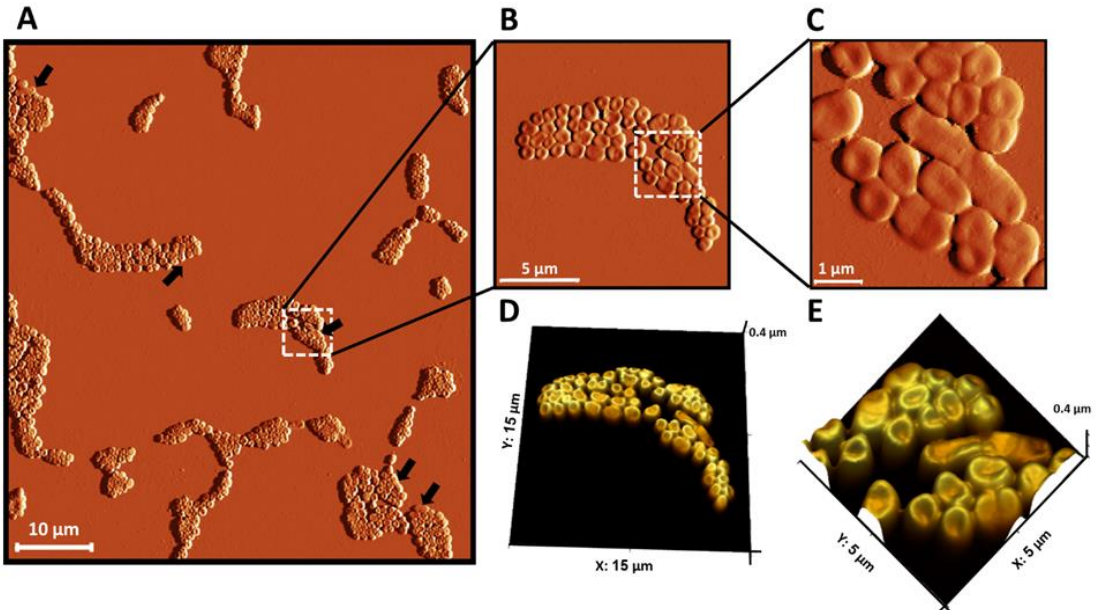

Figure 2. Morphology of A. baumannii ACICU cells. Coccoid and rod shape of A. baumannii ACICU grown for $14 \mathrm{~h}$ in flask at $37^{\circ} \mathrm{C}$ with shaking. Bacterial cells were harvested, washed twice and diluted to $\mathrm{OD}_{600}=1$ in distilled water. An aliquot of $20 \mu 1$ of the bacterial suspension was deposited on a microscope glass slide and air-dried for $20 \mathrm{~min}$ at room temperature before imaging with AFM. (A) Large-scale image of different bacterial clusters. (B) Zoom up of an individual cluster. (C) Detailed view of individual cells within the cluster. (D, E) 3D renderings of AFM images of a cluster at different magnifications. Black arrows indicate elongated cells. Relative insets of a filamentous cell and the corresponding 3D projections were shown.

We notice that, albeit AFM analysis indicates that A. baumannii ACICU typically features a coccus shape, $4 \mu \mathrm{m}$-long rod-shaped cells were sporadically detected in the logarithmic phase (Figure $2 \mathrm{~A}, 2 \mathrm{~B}$, and $2 \mathrm{C}$ ), in agreement with a previous study reporting a bacillus-like morphology for cells in the logarithmic growth phase (39). From the high magnification 3D rendering of a rod-shaped cell (Figure 2D and 2E), it was possible to observe a lower height and a larger footprint compared to the surrounding coccus cells. The abundance of elongated cells observed in the early-logarithmic growth phase was $<0.3 \%$ only, increasing to $\sim 1.5 \%$ in the latelogarithmic growth phase. Interestingly, rod-shaped cells were not detected in samples collected at $>6 \mathrm{~h}$ incubation, following examination of > 300 cells, suggesting that the elongated shape could be related to an ongoing cell division. 
A
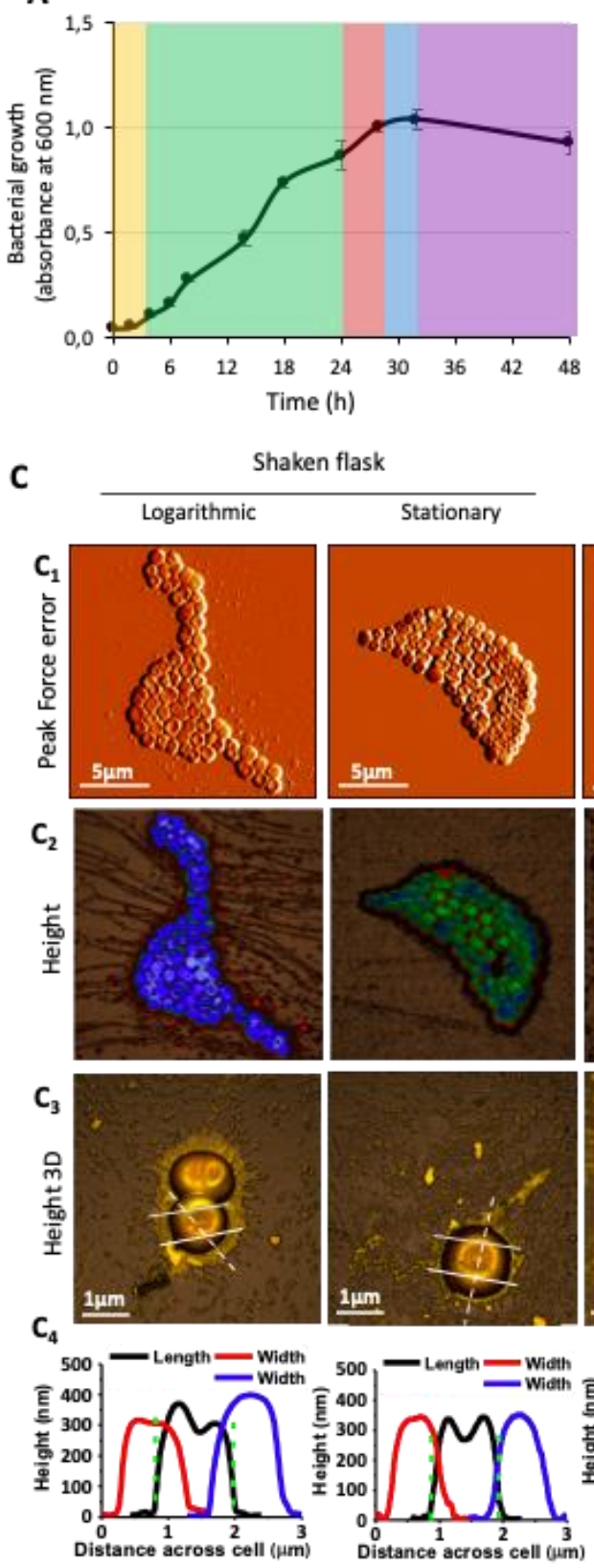

B
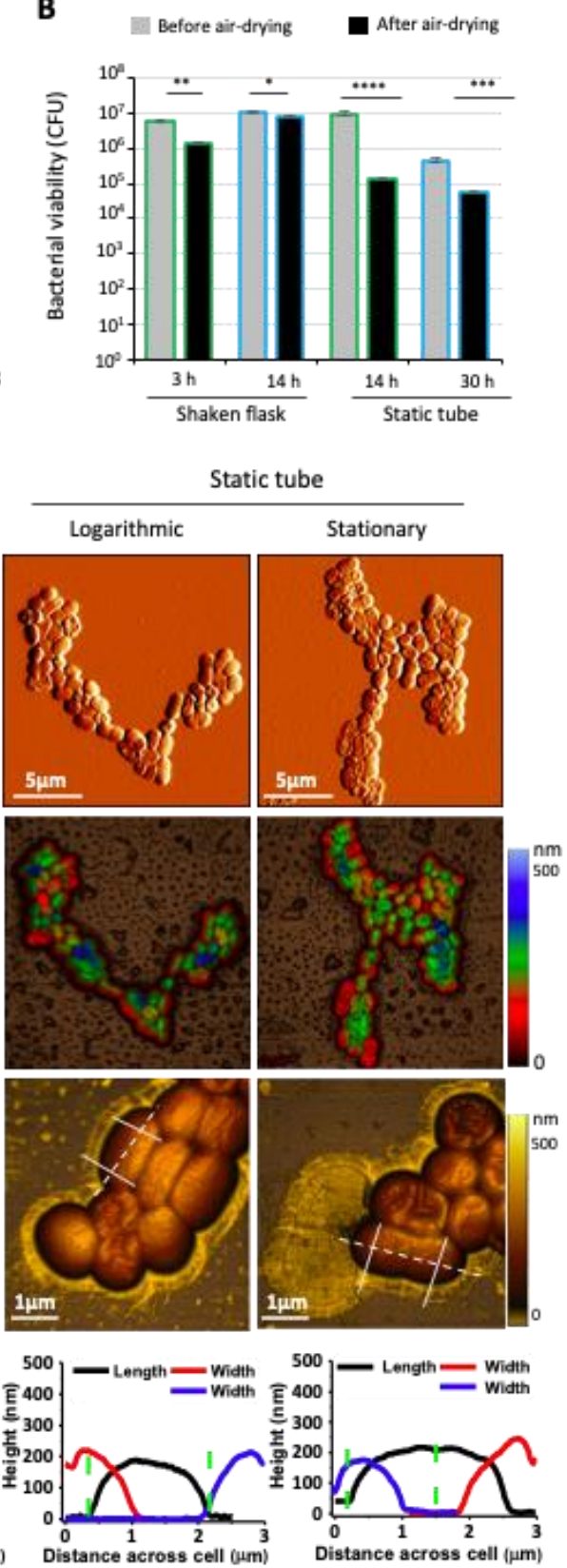

Figure 3. Comparison of growth, cell viability and morphology of A. baumannii ACICU during growth in shaken flasks (SF) and static tube (ST). (A) Growth plot of A. baumannii ACICU cultured in $\mathrm{LB}$ at $37^{\circ} \mathrm{C}$ in static condition. At the indicated time points, bacterial growth was measured as absorbance at $600 \mathrm{~nm}\left(\mathrm{OD}_{600}\right)$. The coloured boxes yellow, green, red, cyan and violet indicate lag, logarithmic, late logarithmic, stationary and death phase, respectively. (B) CFU of A. baumannii ACICU grown in shaking and in static condition. The $\mathrm{CFU}$ was determined before and after air-drying. Colours of the histogram bars outline correspond to the growth phases shown in panel $\mathrm{A}$. (C) AFM imaging of A. baumannii ACICU grown in static and shaking conditions. All of the height images were first-order flattened and set to the same range of height $(0-500 \mathrm{~nm})$ to facilitate comparison between conditions. $\left(\mathrm{C}_{1}\right)$ AFM Peak Force error and $\left(\mathrm{C}_{2}\right)$ height images of A. baumannii ACICU clustered cells. $\left(\mathrm{C}_{3}, \mathrm{C}_{4}\right)$ Large magnification of A. baumannii ACICU single cells and corresponding cross-sectional profiles. The green dashed lines indicate the length of single cell bacteria. Asterisks indicate statistically significant differences between before and after air-drying conditions $(* \mathrm{P}<0.05 ; * * \mathrm{P}<0.01 ; * * * \mathrm{P}<0.001 ; * * * * \mathrm{P}<0.0001)$.

Oxygen deprivation influences desiccation tolerance and cell morphology

Bacterial cell morphology can be influenced by a number of factors like nutrient availability, temperature, light, $\mathrm{pH}$, relative humidity, salt concentration, and oxygenation (40, 41). Although A. baumannii is a strictly aerobic bacterium (42), it can occasionally be faced with low oxygen concentrations, $e$. g. during biofilm-mode growth in vivo (43). Therefore, we wondered whether microaerophilic cultural conditions could influence A. baumannii resistance to desiccation and/or cell morphology (35). 
To this aim, the effects of oxygen deprivation on the growth of $A$. baumannii ACICU has been mimed by inoculating cells in a tube containing $1 / 3$ atmospheric oxygen/cultural medium volume ratio and incubated in static conditions. Since A. baumannii is a nonmotile bacterium, the static condition causes the bacteria to settle at the bottom of the tube, minimizing oxygen availability (44). The effect of static tube (ST) growth condition on A. baumannii ACICU desiccation tolerance and morphology was then compared with those determined for aerated (SF) cultures.

To this purpose, A. baumannii ACICU growth in ST was monitored for up to $48 \mathrm{~h}$ (Figure 3A), and compared with the SF condition (Figure 1A). Cultivation in ST produced an overall slowdown of growth rate, with a clear delay of all growth phases: lag ( $\mathrm{t}_{\text {growth }}<3 \mathrm{~h}$ ), early logarithmic $\left(3 \mathrm{~h}<\mathrm{t}_{\text {growth }}<24 \mathrm{~h}\right.$ ), late logarithmic $\left(24 \mathrm{~h}<\mathrm{t}_{\text {growth }}<26 \mathrm{~h}\right)$, stationary $\left(26 \mathrm{~h}<\mathrm{t}_{\text {growth }}<32 \mathrm{~h}\right)$, and death ( $t_{\text {growth }}>32 \mathrm{~h}$ ) phases. Moreover, the maximum of cell density reached under ST conditions was $\sim 1 / 5 \times$ that reached in SF $\left(\mathrm{OD}_{600}=1.0 \mathrm{vs} . \mathrm{OD}_{600}=5.0\right.$, respectively $)$. Furthermore, the logarithmic growth phase duration $\sim 5 \times$ longer in ST than in SF. Then, the impact of oxygen availability on A. baumannii ACICU desiccation resistance was investigated by comparing the bacterial viability before and after drying between SF and ST cultures (Figure 3B). An overall increased susceptibility to desiccation was observed for ST cells, compared with SF cells, and this effect was evident irrespective of the growth stage (exponential or stationary; Figure 3B). At the exponential phase, the extent of desiccationdependent cell death was $c a$. two log reduction in ST and < one log in SF. Likewise, desiccation had nearly no effect on SF cells at the stationary phase, while it caused one log reduction of ST cells. These results indicate that low oxygen availability increases the susceptibility to desiccation of A. baumannii ACICU.

To extend the investigation of the oxygen availability effect on cell morphology, A. baumannii ACICU cells from SF and ST conditions were imaged by AFM during the logarithmic and stationary growth phases (Figure 3C). A. baumannii ACICU cells grown in a low-oxygen condition (ST) displayed a rod-shaped morphology (Figure 3C $\mathrm{C}_{1}$ ). Furthermore, the diplococcus structure of the A. baumannii ACICU, appearing as two joined cells, and cells with pronounced damage in their outer membranes were frequently detected in both growth phases of the ST condition (Figure $3 \mathrm{C}_{1}$ ).

On the other hand, investigation of AFM height images (Figure $3 \mathrm{C}_{2}$ ) revealed that the cells which were grown in a low-oxygen condition (ST) displayed reduced height as compared to SF cells. A height reduction was also observed in cells harvested from the stationary phase in SF (Figure 1C), which were characterized by a maximum survival rate after desiccation (Figure 3B). Therefore, the height variation is not predictive of desiccation tolerance.

Table 2. Cellular dimensions of A. baumannii ACICU grown in static tube (ST).

\begin{tabular}{|c|c|c|c|c|c|}
\hline \multirow{2}{*}{ Growth phase } & \multicolumn{5}{|c|}{ Cellular dimensions } \\
\hline & Length $(\mu \mathrm{m})$ & Width $(\mu \mathrm{m})$ & Height $(\mu \mathrm{m})$ & Volume $\left(\mu \mathrm{m}^{3}\right)$ & Surface/Volume $\left(\mu \mathrm{m}^{-1}\right)$ \\
\hline Logarithmic & $1.73 \pm 0.22$ & $1.11 \pm 0.13$ & $0.244 \pm 0.31$ & $0.275 \pm 0.04$ & $5.091 \pm 0.63$ \\
\hline Stationary & $1.67 \pm 0.30$ & $1.05 \pm 0.12$ & $0.232 \pm 0.25$ & $0.263 \pm 0.05$ & $5.498 \pm 1.28$ \\
\hline
\end{tabular}



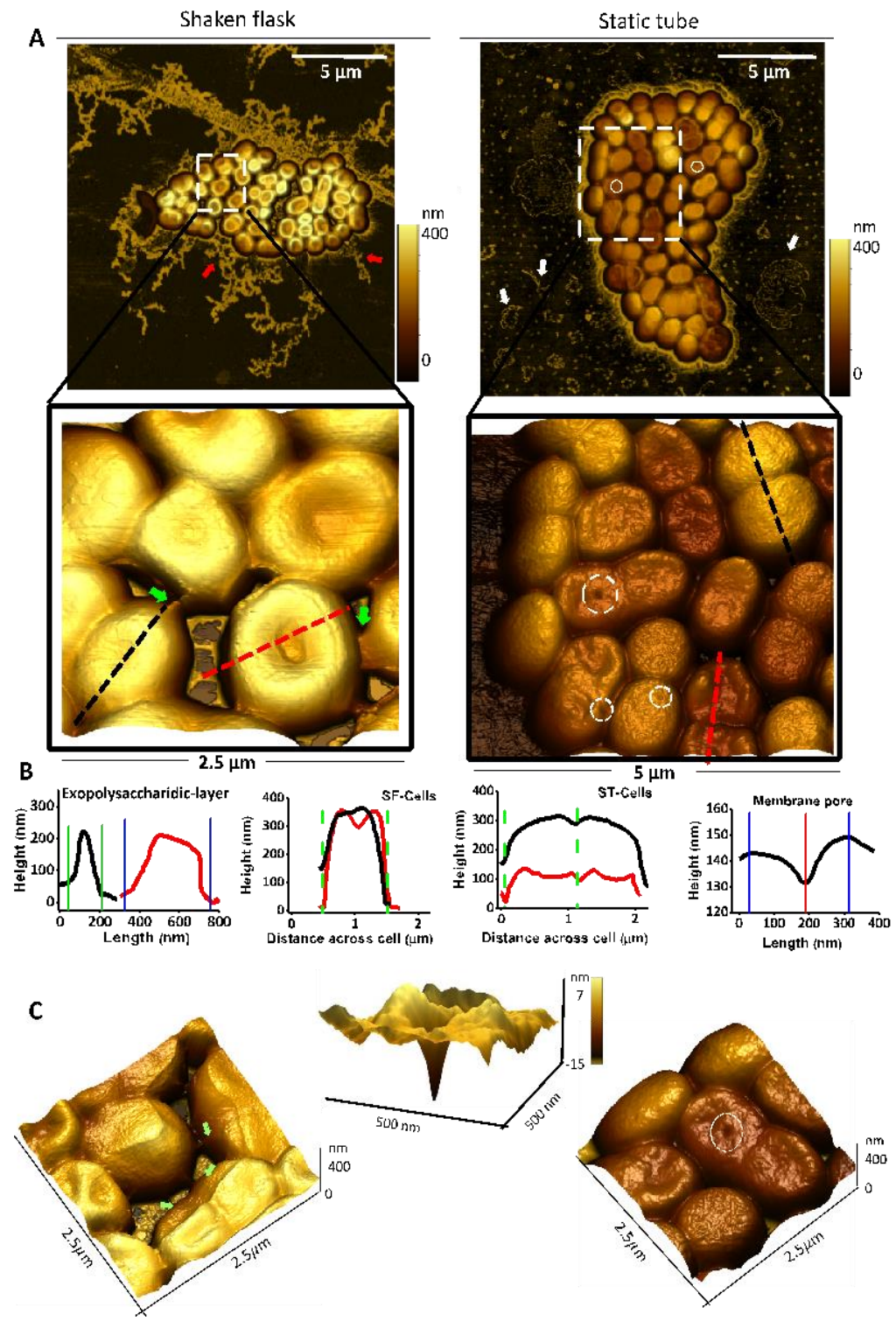

Figure 4. Surface morphology of A. baumannii ACICU cells after growth under shaking and static conditions. A. baumannii ACICU cells harvested from logarithmic phase were imaged by AFM after desiccation (A) and the scale bar was set from 0 to $400 \mathrm{~nm}$ in all images. The red and white arrows denote the extracellular materials in shaken flask and release of DNA in the static tube, respectively. The insets represent high resolution $(704 \times 704$ pixels/line) images of A. baumannii ACICU cells. Green arrows and white dotted circles show the presence of an extracellular matrix, presumably an exopolysaccharide layer, on the cell surface in SF and membrane pores in ST, respectively. The red and black dashed lines show the cross-sectional height of intact and collapsed A. baumannii ACICU cells in both conditions. Panel (B) from left to right displays the corresponding profiles of extracellular matrix, the surface structure of A. baumannii ACICU cells in SF, ST and cell membrane pores, respectively. Large magnification (3D) AFM images of A. baumannii ACICU surface characteristics (C) in SF and ST conditions along with 3D images of membrane pores are illustrated.

A more detailed analysis of A. baumannii ACICU morphology under different growth conditions is provided by the magnified AFM images in Figure $3 \mathrm{C}_{3}$. Bacterial cells in the $\mathrm{SF}$ condition displayed a coccus morphology with a smooth surface. On the contrary, cells grown in ST condition showed a rod-shape with an uneven surface. Cross-sectional lines in the bottom panels of Figure $3 \mathrm{C}_{4}$ clearly highlight a height decrease and a sizable increase in length for the A. baumannii ACICU cells grown in low-oxygen conditions (ST). Furthermore, in the ST condition, most of the cells do not display the central depression typical of the cell grown in $\mathrm{SF}$ condition (Figure 3C and 4A, 4B). Additionally, the morphology and structure of the cells remained relatively unchanged in the different growth phases of each condition. These findings suggest that cell morphology is markedly influenced by oxygen availability rather than the growth phase.

To better characterize A. baumannii ACICU cells grown in ST, the length, width, height, volume, and surface/volume ratio were calculated for more than 30 air-dried cells harvested from logarithmic and stationary growth phases (Table 2). ST cells are characterized by a $\sim 1.6 \mathrm{~L} / \mathrm{W}$ ratio in both logarithmic and stationary phases. Moreover, no significant differences for all the calculated parameters were observed between ST cells from the logarithmic and stationary phase. Of note, ST cells grown under 
oxygen-deprivation condition appeared to be $c a 1.5$-fold longer and 0.7-fold thinner than those cultured in SF (Table 1). It must be pointed out that nearly all the measured ST cells should be considered dead, given the dramatic reduction of cell viability observed after desiccation (Figure 3B). Interestingly, ST cells had a larger footprint and surface to volume ratio compared with SF cells, corroborating our observations that a larger cell relative footprint is related to a decrease in viability.

Since morphological differences were observed depending on the oxygenation level, AFM comparative analysis at higher resolution was performed on SF and ST cells (Figure 4). To this aim, different fields of view, from $15 \mu \mathrm{m}$ to $500 \mathrm{~nm}$ images were acquired, emphasizing the surface and cellular characteristics of bacteria. As shown in Figure 4A, we observed aggregation of extracellular material around both SF (see red arrows) and ST cells (see white arrows). On the basis on morphological analysis previously reported in literature $(45,46)$ and the height obtained from the line profiles displayed in Fig. S2 (Supporting Information), the material around ST cells (white arrows) is compatible with DNA. We argue that this material is probably being leaked out from the damaged cells. Conversely, the morphology of the extracellular materials around SF cells (red arrows) is completely different from that of DNA (white arrows). Despite we cannot univocally identify the material around the SF cells, we can rule out that it originates from the growth medium, since the bacterial cells were washed twice with distilled water before AFM analysis (see Experimental section).

The high- resolution images in the inset revealed the presence of a smooth layer covering the SF bacteria (inset of Figure 4A, green arrows). This layer, which is absent in ST bacteria, could be composed of exopolysaccharides that could play an osmoprotectant role by retaining water in the local microenvironment, protecting bacterial cells from dehydration, and enhancing the survival in harsh environments $(34,47)$. AFM profile analy sis revealed that the thickness of these layers, occasionally observed in contact regions between cells (green arrows), was in the 100- $400 \mathrm{~nm}$ range (Figure 4B). On the other hand, the surface of ST was characterized by visible short-wavelength ripples and also relatively large pores (Figure 4A, see dashed circles) (48). These pores reflect major alterations in membrane integrity caused by desiccation. The pore average depth and diameter measured 20$30 \mathrm{~nm}$ (Figure 4B). These holes were large enough to allow the leakage of intracellular material, including that observed on the glass slide in the neighborhood of some cell clusters (Figure 4A, white arrows). The 3D rendering of the AFM provided in Figure $4 \mathrm{C}$ further evidences the negative part, i.e. the dip in the membrane of A. baumannii ACICU cells grown in ST condition.

Moreover, the black and red dashed lines over the cells in the inset of Figure 4A indicate the intact and collapsed bacterial cells, respectively, in both growth conditions. The corresponding profiles in Panel B clearly demonstrate the deformation of the cells. We carried out local surface roughness analysis of individual cell membranes for both ST and SF growth conditions, performing each measurement at least forty different cells. For ST cells, the nearly identical surface roughness of $1.0 \pm 0.2 \mathrm{~nm}$ and $0.9 \pm 0.2 \mathrm{~nm}$ was observed for the logarithmic and stationary phase, respectively (Figure S1). This finding is in line with an earlier study on the $A$. baumannii type strain ATCC $19606^{\mathrm{T}}$, showing that the membrane roughness was invariant for cells across all growth phases (4). Similarly, in the SF conditions, we measured a local surface roughness of $1.5 \pm 0.3 \mathrm{~nm}$ and $1.1 \pm 0.3 \mathrm{~nm}$ for the logarithmic and stationary phase, respectively. The ST membrane roughness values were measured to be slightly lower than those observed in the SF condition seems, at a first glance of Fig. 4, counterintuitive. In fact, despite being particularly eye-catching in the images due to their short wavelength and spatial correlation length, they have a relatively low height amplitude (see Fig. S1).

\section{- CONCLUSIONS}

The peculiar ability of A. baumannii to overcome the desiccation stress certainly contributes to the adaptive success of this bacterium in the nosocomial environment. In the present work, we demonstrated that desiccation tolerance and cellular shape and dimension are strongly influenced by the growth phases and by oxygen availability. A. baumannii ACICU cells featured a predominant coccoidal morphology at all growth phases, although rare rod-shaped elements were detected in the logarithmic phase. Moreover, A. baumannii ACICU cells in the early-logarithmic phase appeared more susceptible to desiccation than those in the stationary phase. Intriguingly, A. baumannii cells in the stationary phase display the smallest surface/volume ratio, indicating a possible negative correlation between cell dimension and desiccation resistance. Regarding the effect of oxygen on cell shape and desiccation resistance, a significant decrease in cell height and volume, together with a significant reduction of survival rates, were observed for $A$. baumannii cells cultured in a micro-aerobic environment. AFM measurements revealed that growth under microaerobic conditions causes the frequent formation of membrane pores (likely responsible for intracellular material leakage) and an increase of surface RMS roughness, which represents a novel cellular marker of environmental fitness decline, after exposure to dry conditions. More importantly, our results correlate morphology alteration with desiccation tolerance of $A$. baumannii ACICU cells, suggesting that the rod-shaped morphotype of A. baumannii ACICU, occurred under low-oxygen condition, is highly susceptible to desiccation compared with the coccoid shape. In conclusion, this work provides a preliminary description of some functional and morphological changes occurring in A. baumannii during desiccation under condition mimicking those encountered in the hospital environment and paves the way for the use of AFM as a tool for the investigation of the adaptive response to dehydration, or other environmental stresses.

\section{ACKNOWLEDGMENT}

This work was supported by the Excellence Departments grant from the Italian Ministry of Education, University and Research (MIUR, Italy) (Art. 1, commi 314-337 Legge 232/2016) to the Department of Science, Roma Tre University, and by the PRIN 2017 grant protocol 20177J5Y3P from MIUR to P.V. We acknowledge the technical support of the LIME laboratory at Roma Tre University.

\section{ASSOCIATED CONTENT}

Supporting Information. Surface characterization (normal distribution and histogram of surface roughness) for ST cells in both logarithmic and stationary growth phases. Line profiles showing the height of the extracellular material observed around ST cells.

\section{- AUTHOR INFORMATION}


Corresponding Author

Email: giovanni.capellini@uniroma3.it

Notes

The authors declare no conflict of interest

\section{- REFERENCES}

(1) Van Teeseling, M. C. F.; de Pedro, M. A.; Cava, F. Determinants of Bacterial Morphology: From Fundamentals to Possibilities for Antimicrobial Targeting. Front. Microbiol. 2017, 8. https://doi.org/10.3389/fmicb.2017.01264.

(2) Yang, D. C.; Blair, K. M.; Salama, N. R. Staying in Shape: The Impact of Cell Shape on Bacterial Survival in Diverse Environments. Microbiol. Mol. Biol. Rev. 2016, 80 (1), 187203. https://doi.org/10.1128/MMBR.00031-15.

(3) Young, K. D. The Selective Value of Bacterial Shape. Microbiology and Molecular Biology Reviews 2006, 70(3), 660-703. https://doi.org/10.1128/mmbr.00001-06.

(4) Soon, R. L.; Nation, R. L.; Hartley, P. G.; Larson, I.; Li, J. Atomic Force Microscopy Investigation of the Morphology and Topography of Colistin-Heteroresistant Acinetobacter baumannii Strains as a Function of Growth Phase and in Response to Colistin Treatment. Antimicrobial Agents and Chemotherapy 2009, 53 (12), 4979-4986. https://doi.org/10.1128/AAC.00497-09.

(5) Soon, R. L.; Nation, R. L.; Harper, M.; Adler, B.; Boyce, J. D.; Tan, C.-H.; Li, J.; Larson, I. Effect of Colistin Exposure and Growth Phase on the Surface Properties of Live Acinetobacter baumannii Cells Examined by Atomic Force Microscopy. International Journal of Antimicrobial Agents 2011, 38 (6) 493-501. https://doi.org/10.1016/j.ijantimicag.2011.07.014.

(6) Htoo, H. H.; Brumage, L.; Chaikeeratisak, V.; Tsunemoto, H.; Sugie, J.; Tribuddharat, C.; Pogliano, J.; Nonejuie, P. Bacterial Cytological Profiling as a Tool To Study Mechanisms of Action of Antibiotics That Are Active against Acinetobacter baumannii. Antimicrobial Agents and Chemotherapy 2019, 63 (4). https://doi.org/10.1128/AAC.02310-18.

(7) Dijkshoorn, L.; Nemec, A.; Seifert, H. An Increasing Threat in Hospitals: Multidrug-Resistant Acinetobacter baumannii. Nat. Rev. Microbiol. 2007, $5 \quad$ (12), 939-951. https://doi.org/10.1038/nrmicro1789.

(8) Peleg, A. Y.; Seifert, H.; Paterson, D. L. Acinetobacter baumannii: Emergence of a Successful Pathogen. Clinical microbiology reviews https://doi.org/10.1128/CMR.00058-07.

(9) Rice, L. B. Federal Funding for the Study of Antimicrobial Resistance in Nosocomial Pathogens: No ESKAPE. J. Infect. Dis. 2008, 197 (8), 1079-1081. https://doi.org/10.1086/533452.

(10) Wendt, C.; Dietze, B.; Dietz, E.; Ruden, H. Survival of Acinetobacter baumannii on Dry Surfaces. J. Clin. Microbiol. 1997, $35 \quad$ (6), 1394-1397. https://doi.org/10.1128/JCM.35.6.1394-1397.1997.

(11) Jawad, A.; Seifert, H.; Snelling, A. M.; Heritage, J.; Hawkey, P. M. Survival of Acinetobacter baumannii on Dry Surfaces: Comparison of Outbreak and Sporadic Isolates. J. Clin. Microbiol. 1998, $36 \quad$ (7), 1938-1941. https://jcm.asm.org/content/36/7/1938.

(12) Giannouli, M.; Antunes, L. C. S.; Marchetti, V.; Triassi, M.; Visca, P.; Zarrilli, R. Virulence-Related Traits of Epidemic Acinetobacter baumannii Strains Belonging to the International Clonal Lineages I-III and to the Emerging Genotypes ST25 and ST78. BMC Infect. Dis. 2013, 13, 282. https://doi.org/10.1186/1471-2334-13-282.

(13) Bergogne-Berezin, E.; Towner, K. J. Acinetobacter Spp. as Nosocomial Pathogens: Microbiological, Clinical, and Epidemiological Features. Clin. Microbiol. Rev. 1996, 9 (2), 148-165. https://doi.org/10.1128/CMR.9.2.148.
(14) Antunes, L. C. S.; Visca, P.; Towner, K. J. Acinetobacter baumannii: Evolution of a Global Pathogen. Pathog Dis 2014, 71 (3), 292-301. https://doi.org/10.1111/2049-632X.12125.

(15) Harding, C. M.; Hennon, S. W.; Feldman, M. F. Uncovering the Mechanisms of Acinetobacter baumannii Virulence. Nat. Rev. Microbiol. $\quad 2018, \quad 16 \quad$ (2), 91-102. https://doi.org/10.1038/nrmicro.2017.148.

(16) Zeidler, S. \& Muller, V. The Role of Compatible Solutes in Desiccation Resistance of Acinetobacter baumannii. Microbiologyopen 2019, 8 (5), e00740. https://doi.org/10.1002/mbo3.740.

(17) Zeidler, S. \& Muller, V. Coping with Low Water Activities and Osmotic Stress in Acinetobacter baumannii: Significance, Current Status and Perspectives. Environ. Microbiol. 2019, 21 (7), 2212-2230. https://doi.org/10.1111/1462-2920.14565.

(18) Iacono, M.; Villa, L.; Fortini, D.; Bordoni, R.; Imperi, F.; Bonnal, R. J. P.; Sicheritz-Ponten, T.; De Bellis, G.; Visca, P.; Cassone, A.; et al. Whole-Genome Pyrosequencing of an Epidemic Multidrug-Resistant Acinetobacter baumannii Strain Belonging to the European Clone II Group. Antimicrob. Agents Chemother. 2008, 52 (7), 2616-2625. https://doi.org/10.1128/AAC.01643-07.

(19) Khursigara, C. M.; Koval, S. F.; Moyles, D. M.; \& Harris, R. J. Inroads through the Bacterial Cell Envelope: Seeing Is Believing. Canadian Journal of Microbiology 2018, 64 (9), 601-17. https://doi.org/10.1139/cjm-2018-0091

(20) Scheuring, S. \& Dufrene, Y. F. Atomic Force Microscopy: Probing the Spatial Organization, Interactions and Elasticity of Microbial Cell Envelopes at Molecular Resolution. Mol. Microbiol. 2010, 75 (6), 1327-1336. https://doi.org/10.1111/j.1365-2958.2010.07064.x.

(21) Alessandrini, A. \& Facci, P. AFM: A versatile tool in biophysics. Measurement Science and Technology 2005, 16(6). https://doi.org/10.1088/0957-0233/16/6/R01.

(22) Dufrene, Y. F. Atomic force microscopy in microbiology: new structural and functional insights into the microbial cell surface. $\begin{array}{lllll}m \text { mio } & \mathbf{2 0 1 4}, & 5 & \text { (4), } & \text { e1363-14. }\end{array}$ https://doi.org/10.1128/mBio.01363-14.

(23) Dufrene, Y. F.; Ando, T.; Garcia, R.; Alsteens, D.; MartinezMartin, D.; Engel, A.; Gerber, C.; Muller, D. J. Imaging modes of atomic force microscopy for application in molecular and cell biology. Nature Nanotechnology 2017, 12, 295-307. https://doi.org/10.1038/nnano.2017.45.

(24) Chen, C.; Li, G.; Cui, X.; Chen, J.; Yu, Q.; Zong, C.; Zhao, Y.; $\mathrm{Xu}$, M.; Zhou, S.; Xu, H. Mechanistic Investigation of a SelfAssembling Peptide against Escherichia Coli. Langmuir 2020, 36 (33), 9800-9809. https://doi.org/10.1021/acs.langmuir.0c01311.

(25) Xiao, J.; Dufrene, Y. F. Optical and force nanoscopy in microbiology. Nature Microbiology 2016, 1 (11), 16186. https://doi.org/10.1038/nmicrobiol.2016.186.

(26) Viljoen, A.; Foster, S. J.; Fantner, G. E.; Hobbs, J. K.; Dufrene, Y. F. Scratching the Surface: Bacterial Cell Envelopes at the Nanoscale. $\quad$ mBio $\quad 2020, \quad 11$ https://doi.org/10.1128/mBio.03020-19.

(27) Deliorman, M.; Gordesli Duatepe, F. P.; Davenport, E. K.; Fransson, B. A.; Call, D. R.; Beyenal, H.; Abu-Lail, N. I. Responses of Acinetobacter baumannii Bound and Loose Extracellular Polymeric Substances to Hyperosmotic Agents Combined with or without Tobramycin: An Atomic Force Microscopy Study. Langmuir 2019, 35 (27), 9071-9083. https://doi.org/10.1021/acs.langmuir.9b01227.

(28) Kuo, H.-Y.; Chao, H.-H.; Liao, P.-C.; Hsu, L.; Chang, K.-C.; Tung, C.-H.; Chen, C.-H.; Liou, M.-L. Functional Characterization of Acinetobacter baumannii Lacking the RNA Chaperone Hfq. Front. Microbiol. 2017, 8. https://doi.org/10.3389/fmicb.2017.02068.

(29) Dubrovin, E. V.; Popova, A. V.; Kraevskiy, S. V.; Ignatov, S. G.; Ignatyuk, T. E.; Yaminsky, I. V.; Volozhantsev, N. V. Atomic Force Microscopy Analysis of the Acinetobacter 
baumannii Bacteriophage AP22 Lytic Cycle. PLoS One 2012, 7 (10). https://doi.org/10.1371/journal.pone.0047348.

(30) Dubrovin, E. V.; Voloshin, A. G.; Kraevsky, S. V.; Ignatyuk, T. E.; Abramchuk, S. S.; Yaminsky, I. V.; Ignatov, S. G. Atomic Force Microscopy Investigation of Phage Infection of Bacteria. Langmuir 2008, $24 \quad$ (22), 13068-74. https://doi.org/10.1371/journal.pone.0047348.

(31) James, S. A.; Hilal, N.; Wright, C. J. Atomic Force Microscopy Studies of Bioprocess Engineering Surfaces - Imaging, Interactions and Mechanical Properties Mediating Bacterial Adhesion. Biotechnol $J \quad \mathbf{2 0 1 7}, \quad 12 \quad$ (7). https://doi.org/10.1002/biot.201600698.

(32) Bertani, G. Lysogeny at mid-twentieth century: P1, P2, and other experimental systems. J. Bacteriol. 2004, 186:595-600. https://doi.org/10.1128/jb.186.3.595-600.2004.

(33) Necas, D., \& Klapetek, Gwyddion: an open-source software for SPM data analysis, Open Physics, 2012, 10(1), 181-188. https://doi.org/10.2478/s11534-011-0096-2

(34) Farrow, J. M.; Wells, G.; Pesci, E. C. Desiccation Tolerance in Acinetobacter baumannii Is Mediated by the Two-Component Response Regulator BfmR. PLoS ONE 2018, 13 (10), e0205638. https://doi.org/10.1371/journal.pone.0205638.

(35) Kysela, D. T.; Randich, A. M.; Caccamo, P. D.; Brun, Y. V. Diversity Takes Shape: Understanding the Mechanistic and Adaptive Basis of Bacterial Morphology. PLOS Biology 2016 $14 \quad$ (10), e1002565. https://doi.org/10.1371/journal.pbio.1002565.

(36) Mampallil, D. \& Eral, H. B. A Review on Suppression and Utilization of the Coffee-Ring Effect. Adv Colloid Interface Sci 2018, 252, 38-54. https://doi.org/10.1016/j.cis.2017.12.008.

(37) Madigan, M.; Martinko, J. ; Parker, J. Brock Biology of Microorganisms, Prentice Hall, Upper Saddle River, NJ 1997.

(38) Nystrom, T. Stationary-Phase Physiology. Annu. Rev. Microbiol. $\quad \mathbf{2 0 0 4}, \quad 58, \quad 161-181$ https://doi.org/10.1146/annurev.micro.58.030603.123818.

(39) Navarro Llorens, J. M.; Tormo, A.; Martinez-Garcia, E. Stationary Phase in Gram-Negative Bacteria. FEMS Microbiol Rev 2010, 34 (4), 476-495. https://doi.org/10.1111/j.15746976.2010.00213.x
(40) Wilkinson, J. F.; Duguid, J. P. The Influence of Cultural Conditions on Bacterial Cytology. In International Review of Cytology 2008. https://doi.org/10.1016/S0074-7696(08)627448

(41) Donelli, G.; Matarrese, P.; Fiorentini, C.; Dainelli, B.; Taraborelli, T.; Di Campli, E.; Di Bartolomeo, S.; Cellini, L. The Effect of Oxygen on the Growth and Cell Morphology of Helicobacter pylori. FEMS Microbiol. Lett. 1998, 168 (1), 915. https://doi.org/10.1111/j.1574-6968.1998.tb13248.x.

(42) Howard, A.; O’Donoghue, M.; Feeney, A.; Sleator, R. D. Acinetobacter baumannii. Virulence 2012, 3 (3), 243-250. https://doi.org/10.4161/viru.19700.

(43) Eze, E. C., Chenia, H. Y., \& El Zowalaty, M. E. Acinetobacter baumannii biofilms: effects of physicochemical factors, virulence, antibiotic resistance determinants, gene regulation, and future antimicrobial treatments. Infection and drug resistance, $\mathbf{2 0 1 8}, \quad 11, \quad 2277-2299$. https://doi.org/10.2147/IDR.S169894

(44) Somerville, G. A.; Proctor, R. A. Cultivation Conditions and the Diffusion of Oxygen into Culture Media: The Rationale for the Flask-to-Medium Ratio in Microbiology. BMC Microbiol 2013, 13, 9. https://doi.org/10.1186/1471-2180-13-9.

(45) Lyubchenko, Y. L.; Gall, A. A.; Shlyakhtenko, L. S Visualization of DNA and Protein-DNA Complexes with Atomic Force Microscopy. Methods Mol Biol 2014, 1117, 367384. https://doi.org/10.1007/978-1-62703-776-1_17.

(46) Lyubchenko, Y. L. Preparation of DNA and Nucleoprotein Samples for AFM Imaging. Micron 2011, 42 (2), 196-206. https://doi.org/10.1016/j.micron.2010.08.011.

(47) Chang, W.-S.; van de Mortel, M.; Nielsen, L.; Nino de Guzman, G.; Li, X.; Halverson, L. J. Alginate Production by Pseudomonas putida Creates a Hydrated Microenvironment and Contributes to Biofilm Architecture and Stress Tolerance under Water-Limiting Conditions. J. Bacteriol. 2007, 189 (22), 8290 8299. https://doi.org/10.1128/JB.00727-07.

(48) Typas, A.; Banzhaf, M.; Gross, C. A.; Vollmer, W. From the Regulation of Peptidoglycan Synthesis to Bacterial Growth and Morphology. Nat Rev Microbiol 2011, 10 (2), 123-136. https://doi.org/10.1038/nrmicro2677. 


\section{Bacterial growth conditions}
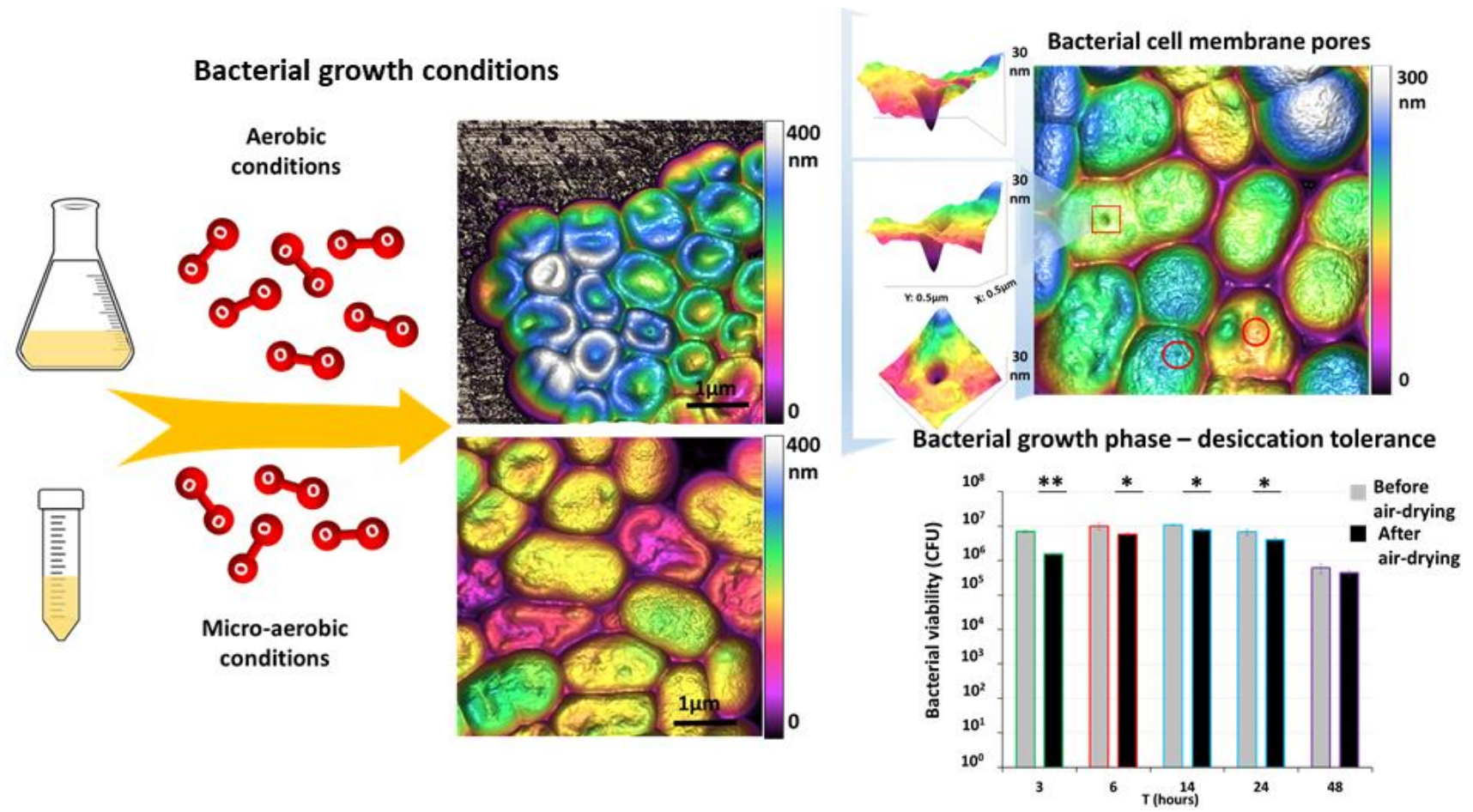